Bioscientia Medicina: Journal of Biomedicine \& Translational Research

Journal Homepage: www.bioscmed.com

\title{
Surgical Site Infection Post Caesarean Section: A Case Report
}

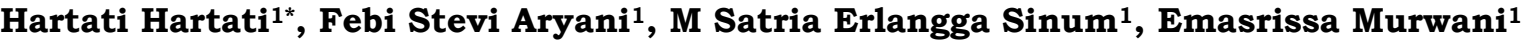

${ }^{1}$ Department of Obstetrics and Gynecology, Faculty of Medicine, Universitas Sriwijaya/Dr. Mohammad Hoesin General Hospital, Palembang, Indonesia

\section{A R T I C L E I N F O}

Keywords:

Antibiotics

Sectio Caesarean

Surgical Site Infection

*Corresponding author:

Hartati Hartati

E-mail address:

drhartatispog@gmail.com

\section{A B S T R A C T}

Background. Surgical site infection (SSI) is a major complication of surgery which can increase morbidity, mortality and cost of hospitality. Incidence of SSI at a healthcare provider/facility reflected a service quality of that institution. Case presentations. A 28-years-old woman, a housewife, P2A1, post-cesarean section 12 days ago. The patient came with the main complaint of weakness and pus in the surgical wound. He complained that his stomach was getting hotter, and clear fluid came out before pus appeared on the surgical wound. Patients also complain of fever, nausea and weakness lately Laboratory investigations Showed hemoglobin $11.9 \mathrm{~g} / \mathrm{dL}$, leukocytes 11,100 $\square / \mathrm{L}$, platelets 601,000. 70.7 MCV fL MCH 24 pg MCHC $32 \mathrm{~g} / \mathrm{dL}$ TIBC $392 \square \mathrm{g} / \mathrm{dL}$ serum Iron $54 \square \mathrm{g} / \mathrm{dL}$ Ferritin $19.8 \mathrm{ng} / \mathrm{mL}$, CRP reactive. Patient was wound dressing and given levofloxacin 500mg three times a day intravascular for three days and continued with ciprofloxacin three times a day oral. Patient was suggested to repeat laboratory tests after therapy to evaluate therapy response. Conclusion: The result showed that time of prophylactic antibiotics can be used to minimize the occurrence of SSI in post-cesarean section patients.

All authors have reviewed and approved the final version of the manuscript.

https://doi.org/10.32539/bsm.v5i5.404

\section{Introduction}

Surgical site infection (SSI) is an infection that occurs in post-surgical patients that can be obtained while the patient is in hospital treatment up to 30 days after surgery. Surgical site infection is a major complication of surgery which can increase morbidity, mortality and cost of hospitality. Incidence of SSI at a healthcare provider/facility reflected a service quality of that institution. WHO reported prevalence of SSI was around $5 \%$ to $34 \% .1,2$

One of the risk factors for SSI is diabetes mellitus and obesity which causes preoperative hyperglycemia. Diabetes mellitus (DM), especially type 2 diabetes, is increasing in prevalence in America, with an estimated $7 \%$, or 20 million people suffering from this disease, with a third of them not realizing that they have DM. The percentage of patients with DM undergoing surgery is high in some types of surgery. One study noted that
$44 \%$ of patients undergoing cardiac surgery had diabetes, whereas $48 \%$ of patients were not diagnosed with diabetes preoperatively. DM is the main predictor that determines the morbidity and mortality of postCABG patients, where about $35 \%$ to $50 \%$ of complications occur in patients with DM. The poor postoperative outcome in patients with DM is believed to be related to the pre-existing complications of chronic hyperglycemia, which include atherosclerotic vascular disease and autonomic peripheral neuropathy. It is very important to carry out a preoperative evaluation of all patients who will undergo surgery to avoid undiagnosed and/or uncontrolled DM cases. ${ }^{3}$

As for the clinical criteria that can be used to define surgical wound infection, namely: there are purulent exudats that come out through surgical wounds, there 
are positive cultures obtained from primary closed surgical wounds, surgical wounds require reopening. This case report aims to present a case of surgical site infection post caesarian operation.

\section{Case Presentation}

A 28-years-old woman, a housewife, P2A1, postcesarean section 12 days ago. The patient came with the main complaint of weakness and pus in the surgical wound. He complained that his stomach was getting hotter, and clear fluid came out before pus appeared on the surgical wound. The patient also complained of fever, nausea and weakness recently.

On physical examination, vital signs were found within normal limits, body mass index was overweight, abdominal examination showed reddened wet wounds and a little pus on the surgical scars. Laboratory investigations Showed hemoglobin $11.9 \mathrm{~g} / \mathrm{dL}$, leukocytes $11,100 \mu / \mathrm{L}$, platelets 601,000. 70.7 MCV fL MCH 24 pg MCHC 32 g / dL TIBC 392 g / dL serum Iron 54 gg / dL Ferritin 19.8 ng / mL, CRP reactive.

The patient was then hospitalized for observation and wound cleaning. Patient was wound dressing and given levofloxacin 500mg three times a day intravascular for three days and continued with ciprofloxacin three times a day oral. Patient was suggested to repeat laboratory tests after therapy to evaluate therapy response.

\section{Follow-up}

\begin{tabular}{|l|l|}
\hline The first day & the main complaints: \\
& Pasien complained of pus in the wounds, pain in the surgical scar. \\
Physical examination: vital signs within normal limits \\
Management: \\
Ringer Lactate IVFD 20 drops per minute \\
Ceftriaxone 3x1 \\
Gentamicin 2x1 \\
Reopening and wound dressing \\
\hline Sixth day of treatment & Subjective complaint: \\
& Reduced pus in the wound and still feels pain in the surgical wound \\
& Physical examination: vital signs in normal limits \\
& Management: \\
& Observation of vital sign \\
& Ringer lactate IVFD 20 drip/minute \\
& Ceftriaxone 3x1 \\
& Metronidazole 3x1 \\
& Sanprima 3x 500 \\
\hline Eighth day of treatment & Subjective complaint: pain in wound after surgery \\
& Physical examination: vital sign within normal limits \\
& Management: \\
& Observation of vital sign \\
& Ringer lactate IVFD 20 drip / minute \\
& Ceftriaxone 3x1 \\
& Metronidazole 3x1 \\
& Sanprima 3x 500 \\
\hline
\end{tabular}

\section{Discussion}

\section{The diagnosis of surgical site infection (SSI)}

Infection surgical wound is an infection that occurs in patients post surgery in the can for patients in hospital treatment until 30 days post surgery. The SSI criteria set by the National Healthcare Safety Network (NHSN) for superficial incisional SSI must meet all of the following criteria: infection occurred within 30 days after the surgical procedure and involved skin and subcutaneous tissue during the operation. Symptoms experienced by the patient were purulent drainage (pus discharge) from the superficial incision and there were specific organisms associated with the postoperative superficial wound (as evidenced by bacterial culture). 4.5

Meanwhile, deep incisional SSI must meet all of the following criteria; Wound infection occurs within 30-90 days postoperatively and involves the deep soft tissues 
of the incision (e.g. fascia and muscle layers). The patient also demonstrates at least one of the following purulent drainage from the deep incision or the presence of an abscess or other evidence of infection in the deep incision detected on histopathological or gross anatomic examination, or imaging tests. In the patient, suppuration occurred on the 12th postoperative day and met the criteria within 30 days of the procedure. Infection in the patient also involves the deeper layers such as the fascia or muscles and there is purulent fluid in the surgical site. ${ }^{6}$

\section{Risk factors for SSI}

The incidence of infection after caesarean section is greater in obese women. Obesity is a risk factor for SSI that has proven difficult to suppress. Often there is not enough time before surgery to significantly reduce the patient's obesity rate. However, evaluation of the presence of diabetes and control of serum glucose levels will minimize the risk of SSI in obese patients. In addition, major surgery is often viewed as a lifechanging event and may motivate patients to adopt a positive diet and other lifestyle. Individualized education and diet management from nutritionists, as well as support from the community trying to lose weight have also shown positive long-term effects. The patient was known to weigh $65 \mathrm{~kg}$ and TB $155 \mathrm{~cm}$ which showed BMI results were 27.1, namely overweight where obesity was one of the factors in the occurrence of SSI in patients.

\section{Measures to prevent the occurrence of SSI}

Several steps involved in reducing the likelihood of the occurrence of SSI based on the guidelines from NICE (National Institute for Health and Clinical Excellence), including: abdominal wall incision, skin incision technique selection in surgery and administration of antibiotics after cesarean section. A caesarean section should be performed using a transverse abdominal incision because this method causes less postoperative pain and a better cosmetic effect than a midline incision. The transverse incision according to Joel Cohen is an option because it is associated with a shorter operating time and reduced postoperative fever morbidity. 3.5

Closure of the abdominal wall at the midline incision is performed by continuous suture using a slow-absorbed thread because this method has a lower incidence of incisional hernia and wound dehiscence compared to the layered closure method. Subcutaneous tissue closure is not routinely performed, except in women who have a subcutaneous fat thickness of more than $2 \mathrm{~cm}$, because subcutaneous tissue closure does not reduce the incidence of SSI. The use of superficial drains should not be used in caesarean section. The use of superficial drains has not been shown to reduce the likelihood of SSI. 6.7

Administration of antibiotics Prior to skin incision, caesarean section will reduce the risk of postoperative maternal infection when compared to prophylactic antibiotics given after skin incision, and it has been shown to have no effect on the baby. Prophylactic antibiotics are recommended for all operations involving hollow organs. Administration of prophylactic antibiotics is known to be the most significant protective factor in reducing the incidence of SSI after caesarean section. Antibiotics should be given preoperatively, ideally within 30 minutes of induction of anesthesia. Adequate antibiotic concentrations in serum and tissue will reduce the risk of bacterial development during the postoperative period. However, the administration of prophylactic antibiotics will not prevent contamination that occurs during surgery due to poor surgical technique. ${ }^{1.5}$

In practice, wide variations are found regarding the route of administration of prophylactic antibiotics. Classen et al demonstrated that the timing of prophylactic antibiotics was very important in preventing SSI. Preoperative prophylactic antibiotics are often not given at the optimal time so that drug concentrations during the operative period do not produce effective results. Guidelines published in the Surgical Infection Prevention Guideline recommend that prophylactic antibiotics should be given 60 minutes before incision and discontinued within 24 hours of surgery. 7

Redisinfection of the skin around the incision site 
prior to skin closure has been reported to reduce the incidence of postoperative SSI. It has also been reported that irrigation with an antibiotic solution at the incision site is safe, does not show any side effects, and is an effective method in reducing infectious morbidity and SSI after caesarean section. The risk of infection persists even after the patient is discharged from the hospital. Medical personnel should educate patients and their families on how to treat surgical wounds, how to recognize signs of an SSI and the importance of reporting these symptoms to their surgeon as the primary care provider.

\section{Conclusion}

Surgical site infection management includes administration of antibiotics and treatment of infected wounds. The choice of surgical technique and treatment of risk factors for wound infection should also be carried out in order to reduce the incidence of surgical site infection after caesarean section.

\section{References}

1. Dessu S, Samuel S, Gebremeskel F, Basazin A, Tariku Z, et al. Determinants of post cesarean section surgical site infection at public hospitals in Dire Dawa administration, Eastern Ethiopia: A case control study. PLoS One. 2021; 16(4): e0250174. https://doi.org/10.1371/journal.pone.02501 $\underline{74}$

2. Ayala D, Tolossa T, Markos J, Yilma MT. Magnitude and factors associated with surgical site infection among mothers underwent cesarian delivery in Nekemte town public hospitals, Western Ethiopia. PLos One. 2021; 16(2):

e0250736.

https://doi.org/10.1371/journal.pone.02507 $\underline{36}$

3. Johnson A, Young D, Reilly J. Caesarean section surgical site infection surveillance. J Hosp Infect. 2006; 64(1): 30-5. https://doi.org/10.1016/j.jhin.2006.03.020

4. Jasim HH, Sulaiman SAS, Khan AH, Dawood OT, Abdulameer $\mathrm{AH}$, et al. Incidence and risk factors of surgical site infection among patients undergoing cesarean section. Clin Med Insight. 2017; 9(1). https://doi.org/10.1177/1179559X17725273

5. De D, Saxena S, Mehta G, Yadav R, Dutta R. Risk factor analysis and microbial etiology of surgical site infections following lower segment caesarian section. Int $\mathrm{J}$ Antibiotic. 2013; 283025.

https://doi.org/10.1155/2013/283025

6. Saeed KBM, Greene RA, Corcoran P, O’Neill SM. Incidence of surgical site infection following caesarean section: a systematic review and meta-analysis protocol. BMJ Open. 2017; 7(1): e013037. http://dx.doi.org/10.1136/bmjopen-2016$\underline{013037}$

7. Gur R, Duggal SD, Rongpharpi SR, Srivastava R, Kumar A, et al. Post Caesarean surgical site infections. Arch Clin Microbiol. 2017; 4(4): 102-9. 